\title{
Coordenação fina e escrita de crianças de 6 a 9 anos nascidas a termo e pré-termo*
}

\section{Fine motor coordination and writing of 6-9 year-old children born preterm and full term}

\author{
Natália Barbosa Coronado Garcia ${ }^{1}$, Lívia de Castro Magalhães ${ }^{2}$, \\ Jair Lício Ferreira Santos ${ }^{3}$
}

http://dx.doi.org/10.11606/issn.2238-6149.v26i2p166-175

Garcia NBC, Magalhães LC, Santos JLF. Coordenação fina e escrita de crianças de 6 a 9 anos nascidas a termo e pré-termo. Rev Ter Ocup Univ São Paulo. 2015 maio-ago.;26(2):166-75.

RESUMO: O objetivo deste trabalho consistiu em investigar a relação entre coordenação fina e qualidade da escrita em escolares nascidos pré-termo e a termo. Quanto ao método, utilizou-se de descrição e comparação entre: grupo pré-termo - 28 escolares, com idade gestacional entre 32 e 36 semanas, peso ao nascer $\leq$ $2.500 \mathrm{~g}$, e o grupo a termo - 28 escolares nascidos com idade gestacional $\geq 37$ semanas, peso ao nascer $\geq 2.500 \mathrm{~g}$, emparelhados por gênero, idade, sala escolar e condição socioeconômica. Os pais dos escolares de ambos os grupos responderam a questionários de desempenho motor e classificação econômica. O desempenho motor e a escrita foram examinados com a Avaliação da Coordenação e Destreza Motora (ACOORDEM) e os professores responderam a questionários para identificar déficits motores (DCDQ-Brasil), de atenção e aprendizagem (EDTAH). Sobre os resultados, constatouse diferença significativa em alguns itens dos testes de escrita e coordenação fina, e pré-termos tenderam a desempenho inferior na escrita, coordenação fina e global e maior probabilidade de déficit de atenção e hiperatividade. Foi encontrada correlação entre o desempenho na escrita e alguns itens motores. Os resultados apontaram mais probabilidade a dificuldades motoras e na escrita entre escolares pré-termo, com implicações para assistência e educação dessas crianças.

DESCRITORES: Prematuro; Escrita manual; Destreza motora; Desempenho psicomotor; Transtornos das habilidades motoras; Terapia ocupacional; Avaliação; Criança.
Garcia NBC, Magalhães LC, Santos JLF. Fine motor coordination and writing of 6-9 year-old children born preterm and full term. Rev Ter Ocup Univ São Paulo. 2015 May-Aug.;26(2):166-75.

\begin{abstract}
Objectives: To investigate the relation between fine motor coordination and the quality of writing in school children born preterm and full term. Method: Description and comparison between the preterm group -28 school children with gestational ages between 32 and 36 weeks, birth weight $\leq 2500 \mathrm{~g}$; and full term group -28 school children born at gestational age $\geq 37$ weeks, birth weight $\geq 2500$ g, paired by gender, age, school class and socioeconomic status. The parents of the school children in both groups completed questionnaires on motor performance and economic classification. Motor performance and writing were assessed by the Motor Coordination and Dexterity Assessment (ACOORDEM), and the teachers completed questionnaires to identify motor cordination difficulties (DCDQ-Brazil), attention, and learning (EDTAH) deficits. Results: There were significant differences in some items of writing and fine coordination tests, and preterm infants tended to underperform in writing, fine and global coordination and were more prone to attention deficit and hyperactivity disorder. A correlation between performance in writing and some motor items was found. The results indicated greater probability of motor and writing difficulties among preterm school children, with implications for their care and education.
\end{abstract}

KEYWORDS: Infant, premature; Handwriting; Motor skills; Psychomotor performance; Motor skills disorders; Occupational therapy; Evaluation; Child.

\footnotetext{
*Artigo originado de dissertação de Mestrado pelo Programa de Pós-Graduação em Terapia Ocupacional da Universidade Federal de São Carlos; Trabalho apresentado (apresentação oral) no XI Congresso Paranaense de Terapia Ocupacional (Realizado em 21 a 23 de agosto de 2014 na UFPR, em Curitiba); Fontes de auxílio: CAPES - bolsa de mestrado da primeira autora.

1. Docente do curso de Terapia Ocupacional da UNIGUAÇU e terapeuta ocupacional em Cascavel, PR. E-mail: natalia_bcoronado@hotmail.com.

2. Professora Titular, Depto. de Terapia Ocupacional da Universidade Federal de Minas Gerais. E-mail: liviacmag@gmail.com.

3. Professor Colaborador Sênior, Depto de Medicina Social- Faculdade de Medicina de Ribeirão Preto. E-mail: jairlfs@fmrp.usp.br.

Endereço para correspondência: Natalia Barbosa Coronado Garcia. Rua Rafael Picolli, 2619, casa 3 - Country, Cascavel, PR. CEP: 85810-010. E-mail: natalia_bcoronado@hotmail.com.
} 
Garcia NBC, et al. Coordenação fina e escrita de crianças de 6 a 9 anos. Rev Ter Ocup Univ São Paulo. 2015 maio/ago.;26(2):166-75.

\section{INTRODUÇÃO}

Dentre amplo conjunto de fatores considerados de risco para o desenvolvimento e aprendizagem da criança, destaca-se o nascimento pré-termo e o muito baixo peso, como fatores de risco biológico, sendo a idade gestacional importante fator determinante para a sobrevida e qualidade de vida do neonato ${ }^{1-2}$. Entende-se como recém-nascido pré-termo ou de nascimento prematuro aquele que ocorre abaixo da $37^{\mathrm{a}}$ semana de gestação.

Apesar das pesquisas mais recentes na área se concentrarem nas consequências da prematuridade em recém-nascidos cada vez mais extremos, alguns estudos vêm abordando aspectos do desenvolvimento em pré-termos tardios, nascidos com idade gestacional inferior, porém mais próxima, a 37 semanas $^{3,4}$. O pré-termo tardio vem sendo apresentado como aquele nascido entre 34 e 36 semanas e seis dias de idade gestacional ${ }^{3-6}$.

Há concordância na literatura quanto à maior suscetibilidade de pré-termos, especialmente aqueles nascidos abaixo de $1.500 \mathrm{~g}$, a problemas no desenvolvimento neurológico, cognitivo e, especialmente, alterações/ morbidades mais sutis, porém clinicamente significativas, como atrasos motores, nas habilidades visomotoras, no desempenho acadêmico durante a pré-escolarização e escolarização, na linguagem e em certos aspectos do comportamento, se comparados aos nascidos a termo $0^{5-9}$.

Entre as alterações associadas ao nascimento pré-termo destaca-se também a maior suscetibilidade a apresentar sinais de Transtorno do Desenvolvimento da Coordenação (TDC) e Transtorno de Déficit de Atenção/ Hiperatividade $(\mathrm{TDA} / \mathrm{H})^{8,10-11}$. Metanálise recente da literatura apontou que escolares de muito baixo peso e muito prematuros têm de cinco a oito vezes maior probabilidade de apresentar dificuldades motoras, mesmo que estas não satisfaçam os critérios para diagnóstico de $\mathrm{TDC}^{8}$.

Apesar de na última década os pré-termos tardios ou moderados terem sidos vistos pela obstetrícia e pela pediatria como clinicamente semelhantes aos bebês nascidos a termo, o nascimento pré-termo tardio ou moderado está fortemente relacionado a morbidades do desenvolvimento neurológico ${ }^{3}$. Bebês nascidos entre 34 e 36 semanas apresentam risco três vezes maior de ter paralisia cerebral, além de riscos mais modestos, porém significativamente aumentados, para atraso no desenvolvimento, retardo intelectual ou ambos, se comparados aos nascidos a termo ${ }^{3}$.

Outros estudos também apontam o insucesso escolar como morbidade frequentemente associada ao nascimento pré-termo, sendo que essas crianças são mais susceptíveis à vulnerabilidade em vários domínios relacionados à prontidão escolar, como saúde e desenvolvimento físico, habilidades socioemocionais, de comunicação e cognitivas $^{12-13}$.

Dentre as habilidades relacionadas ao desempenho escolar, há evidencias de maior propensão para dificuldade de escrita em pré-termos ${ }^{12-14}$, o que seria uma consequência natural do pior desenvolvimento das habilidades sensório-motoras, especialmente coordenação visomotora, que são essenciais para a aquisição da escrita ${ }^{14-16}$.

Embora o défict motor associado à prematuridade possa ter impacto na escrita, foi possível localizar apenas dois estudos que abordam especificamente a questão da escrita na criança nascida pré-termo. Dentre esses artigos, um enfoca aspectos motores relacionados à escrita ${ }^{14}$, enquanto o outro aborda aspectos cognitivos ${ }^{12}$. Observa-se que ainda são necessários novos estudos que investiguem a relação entre prematuridade e desempenho na escrita, com enfoque em aspectos motores nos primeiros anos da escolarização, principalmente no que se refere a pré-termos tardios, sendo que nenhum dos estudos localizados apresenta dados sobre o desempenho de crianças brasileiras.

Assim, o presente estudo, realizado como dissertação de mestrado do Programa de Pós-Graduação em Terapia Ocupacional da Universidade Federal de São Carlos, durante o ano de 2013 com financiamento CAPES, com autorização de Comitê de Ética sob CAEE 12059313.5.0000.5504, teve como objetivo investigar a relação entre coordenação motora fina e qualidade da escrita em escolares nascidos moderada ou tardiamente pré-termo e a termo. A qualidade da escrita e habilidades motoras subjacentes, como a coordenação visomotora e destreza manual, foram comparadas em escolares nascidos pré-termo - moderados a tardios - e a termo.

\section{MÉTODO}

O estudo foi realizado em Boa Esperança do Sul, cidade de pequeno porte da região central do interior paulista, no período de janeiro a julho de 2013. A escolha da cidade deveu-se ao grande número de cidades deste porte $\mathrm{e}$ a sua grande representatividade no contexto populacional do estado e do país. O município apresenta população estimada de 14.356 habitantes, divididos em 12.184 residentes na área urbana e 1.461 na área rural (dados do IBGE de 2010). As taxas de mortalidade infantil no município até 1 ano de idade é de 17,78 para cada mil, com taxas de fecundidade de aproximadamente 2,79 filhos por mulher e expectativa de vida de aproximadamente 70,23 anos, e a taxa de alfabetização é considerada como de $85,65 \%$. 
Garcia NBC, et al. Coordenação fina e escrita de crianças de 6 a 9 anos. Rev Ter Ocup Univ São Paulo. 2015 maio/ago.;26(2):166-75.

A amostra foi composta por dois grupos, sendo um de 28 escolares nascidos prematuramente e 28 escolares nascidos a termo. Para a composição do grupo prétermo foram definidos os seguintes critérios de inclusão: nascimento de 2004 a 2006, idade gestacional $\geq 31$ semanas $\mathrm{e} \leq 37$ semanas, com peso $\leq 2.500 \mathrm{~g}$, estar matriculado na rede regular de ensino na cidade estudada no ano de 2013, ter entre 6 e 9 anos, não apresentar diagnóstico ou sinais de deficiência ou necessidade educacional especial, ter autorização dos pais/responsáveis para participação no estudo (Termo de Consentimento Livre e Esclarecido).

Os escolares do grupo a termo foram selecionados seguindo-se os mesmo critérios para idade, ano de nascimento, autorização dos pais/responsáveis e não ter diagnóstico de deficiência, além de ter nascido com idade gestacional $\geq 37$ semanas, peso ao nascer $>2.500 \mathrm{~g}$ e ser do mesmo gênero, idade, sala de aula e nível socioeconômico de uma das crianças do grupo pré-termo (pareamento dos grupos).

A seleção da amostra iniciou-se pelos escolares pré-termos, sendo realizada busca de todas as crianças com histórico de prematuridade no período estudado, por meio de dados da Declaração de Nascidos Vivos (D.N.) do município. Desta forma, foi possível abranger todas as crianças com histórico de prematuridade do município no período estudado. Após análise das D.N., demais informações residenciais e pessoais das crianças e suas famílias foram buscadas por intermédio do sistema do Cartão Nacional do SUS, postos de saúde e Unidades de Saúde da Família. Foram identificadas 36 crianças que se enquadraram nos critérios de inclusão, porém, após busca domiciliar, a amostra foi reduzida para 28 crianças (no caso de uma criança a mãe não autorizou a participação, uma faleceu, quatro não residiam mais no município e duas apresentaram algum tipo de deficiência intelectual). Desta forma, a amostra compreendeu aproximadamente 100\% dos escolares pré-termos que viviam no município que se enquadravam nos critérios de inclusão.

A amostra do grupo a termos foi composta à medida que os escolares pré-termos eram identificados nas escolas. As professoras auxiliaram na indicação de um a termo de mesma sala de cada pré-termo, que se enquadrasse nos critérios de inclusão e pareamento. Após a indicação do a termo, seus pais/responsáveis foram procurados Somente participaram do estudo os escolares que obtiveram autorização dos pais/responsáveis (TCLE).

Todas as crianças foram avaliadas com uso dos seguintes instrumentos:

Roteiro de entrevista não padronizado: com perguntas sobre o histórico gestacional e pós-gestacional, informações pessoais, educacionais e profissionais dos pais, dinâmica familiar e alguns dados escolares das crianças;
Developmental Coordination Disorder Questionnaire - versão brasileira (DCDQ-Brasil): Questionário de fácil aplicação no qual os pais pontuam o desempenho motor de crianças em situações de vida diária, comparando com outras crianças da mesma idade;

Avaliação da Coordenação e Destreza Motora $A C O O R D E M^{16}$ : Teste padronizado específico para detecção de problemas de coordenação motora em crianças de 4 a 8 anos; ainda em desenvolvimento, mas já conta com estudos de validação para crianças brasileiras ${ }^{16-19}$. O teste é dividido em três partes, sendo que no presente estudo foram aplicados os itens referentes à coordenação e destreza manual e dois questionários (pais e professores) para avaliar o desempenho funcional de crianças em casa e na escola. A avaliação da coordenação e destreza manual inclui dois ítens de escrita - cópia do alfabeto e de sentença curta - pontuados por critérios de qualidade e pelo tempo gasto para a realização.

A ACOORDEM foi utilizada por não haver outros testes padronizados validados para avaliação da escrita em crianças brasileiras e por ser um dos poucos testes com normas de desempenho motor para crianças brasileiras ${ }^{20}$.

Escala de Transtorno de Déficit de Atenção e Hiperatividade - versão para professores (ETDAH): Questionário criado e validado no Brasil para identificar sintomas comportamentais de Transtorno de Déficit de Atenção e Hiperatividade (TDAH) por meio da observação direta do professor no contexto escolar.

Critério de Classificação Econômica Brasil (CCEB): Questionário que permite identificar classes econômicas de acordo com o poder de compra das famílias brasileiras.

$\mathrm{O}$ roteiro de entrevista, os questionários para pais da ACOORDEM, o CCEB e o DCDQ-Brasil foram aplicados com os pais dos escolares no ambiente domiciliar, enquanto que o questionário de professores da ACOORDEM e o ETDAH foram respondidos pelos professores dos escolares. A parte da ACOORDEM correspondente à avaliação da coordenação e destreza motora, incluindo escrita, foi aplicada individualmente no ambiente escolar. Conforme as crianças do grupo pré-termo eram avaliadas, era solicitado à professora que indicasse um escolar a termo, com base nos critérios de inclusão e pareamento. Conforme a indicação das professoras, os pais foram procurados e os demais instrumentos foram aplicados com os escolares a termo, seguindo os mesmos procedimentos.

Para garantir confiabilidade dos dados, a avaliadora foi treinada nos procedimentos de avaliação e todas as testagens foram acompanhadas por uma segunda avaliadora, que registrou os dados de maneira independente, sendo obtido consenso nos casos de discordância. 
Garcia NBC, et al. Coordenação fina e escrita de crianças de 6 a 9 anos. Rev Ter Ocup Univ São Paulo. 2015 maio/ago.;26(2):166-75.

Os resultados descritivos incluem frequências absolutas e relativas para as variáveis categóricas, e para as variáveis quantitativas foram utilizadas medidas de tendência central e dispersão. Foi feita comparação inicial dos grupos, usando teste exato de Fisher, para verificar se o pareamento foi adequado. Para comparação dos grupos nas variáveis em estudo, como a maioria não apresentou característica de normalidade, para as variáveis categóricas foi utilizado o teste exato de Fisher e para as outras variáveis foi utilizado o teste de Mann Whitney. Os questionários foram analisados utilizando o somatório da pontuação dos itens, computado como escore total, ou de subáreas, como previsto para cada instrumento. Os itens de desempenho mais relevantes foram analisados individualmente, sendo que alguns itens do teste de escrita foram agrupados no escore total do teste. Correlação de Spearman foi utilizada para investigar a relação entre os escores totais nas duas provas de escrita (cópia de alfabeto e de sentença) e o desempenho nas provas de coordenação fina e destreza manual da ACOORDEM no grupo pré-termo. Para todas as análises foi considerado o nível de significância de 0,05.

\section{RESULTADOS}

\section{Caracterização da amostra}

Como previsto devido às características dos grupos, teste exato de Fisher identificou diferenças significativas em algumas variáveis (Tabela 1), no entanto, não foi encontrada diferença significativa entre os grupos pré-termo e a termo no que diz respeito aos critérios de pareamento: idade $(\mathrm{p}=0,954)$, ano de nascimento $(p=1,00)$, gênero $(p=1,00)$, renda familiar $(p=0,861)$ e classificação econômica $(\mathrm{p}=0,835)$. Ainda sobre renda familiar, a média encontrada foi de um a três salários mínimos.

Tabela 1 - Caracterização da amostra - dados neonatais por grupo

\begin{tabular}{|c|c|c|c|c|c|c|c|c|c|c|}
\hline & \multicolumn{3}{|c|}{ Pré-termo } & \multicolumn{3}{|c|}{ A termo } & \multicolumn{3}{|c|}{ Total da amostra } & \multirow[b]{2}{*}{$\mathbf{p}^{*}$} \\
\hline & $\mathbf{N}$ & $\%$ & $\begin{array}{c}\text { Média por } \\
\text { grupo }\end{array}$ & $\mathbf{N}$ & $\%$ & $\begin{array}{c}\text { Média } \\
\text { por grupo }\end{array}$ & $\mathbf{N}$ & $\%$ & $\begin{array}{c}\text { Média } \\
\text { total }\end{array}$ & \\
\hline \multicolumn{11}{|c|}{$\begin{array}{l}\text { Idade gestacional (semanas) } \\
\text { (a) }\end{array}$} \\
\hline 32 & 2 & 7,14 & \multirow{6}{*}{34,17} & 0 & 0 & \multirow{6}{*}{$* *$} & 2 & 3,57 & \multirow{6}{*}{$* *$} & \multirow{6}{*}{0,001} \\
\hline 33 & 7 & 25 & & 0 & 0 & & 7 & 12,5 & & \\
\hline 34 & 8 & 28,57 & & 0 & 0 & & 8 & 14,29 & & \\
\hline 35 & 6 & 21,43 & & 0 & 0 & & 6 & 10,71 & & \\
\hline 36 & 5 & 17,86 & & 0 & 0 & & 5 & 8,93 & & \\
\hline $37 />37$ & 0 & 0 & & 28 & 100 & & 28 & 50 & & \\
\hline \multicolumn{11}{|l|}{ Idade(anos) } \\
\hline 6 & 4 & 14,29 & \multirow{4}{*}{7,5} & 3 & 10,71 & \multirow{4}{*}{7,5} & 7 & 12,5 & \multirow{4}{*}{7,5} & \multirow{4}{*}{0,954} \\
\hline 7 & 9 & 32,14 & & 11 & 39,29 & & 20 & 35,71 & & \\
\hline 8 & 12 & 42,86 & & 11 & 39,29 & & 23 & 41,07 & & \\
\hline 9 & 3 & 10,71 & & 3 & 10,71 & & 6 & 10,71 & & \\
\hline \multicolumn{11}{|l|}{ Peso ao nascer (g) } \\
\hline até $2.220 \mathrm{~g}$ & 14 & 50 & \multirow{4}{*}{2078} & 0 & 0 & \multirow{4}{*}{3073} & 14 & 25 & \multirow{4}{*}{2575} & \multirow{4}{*}{0,001} \\
\hline $2.230 \mathrm{a} 2.500 \mathrm{~g}$ & 14 & 50 & & 0 & 0 & & 14 & 25 & & \\
\hline 2.510 a $2.990 \mathrm{~g}$ & 0 & 0 & & 14 & 50 & & 14 & 25 & & \\
\hline $3.000 \mathrm{~g} />3.000 \mathrm{~g}$ & 0 & 0 & & 14 & 50 & & 14 & 25 & & \\
\hline
\end{tabular}

Nota: *Teste exato de Fischer; **médias sem possibilidade de cálculo devido à ausência de informações precisas sobre a IG das crianças do grupo a termo.

Informações sobre acompanhamentos recebidos pelas mães dos recém-nascidos pré-termos indicam que em alguns municípios as mães recebem alta hospitalar sem receber qualquer informação a respeito das possíveis consequências da prematuridade, chegando a um índice de $85,71 \%$; e $96,43 \%$ declararam não terem recebido acompanhamento médico especializadoouparticipadodeprogramasdeacompanhamentoao pré-termo.

Com relação à escolarização das crianças da amostra, 89,29\% da amostra total estudam em escolas públicas, o que corresponde a 25 crianças de cada grupo (pré-termo e a termo), enquanto que apenas 10,71\% estavam cursando primeiro grau em escola particular, correspondendo a três crianças de cada grupo.

A comparação dos dados sobre a dinâmica familiar revelou diferença significativa $(\mathrm{p}=0,001)$, com mais crianças do grupo pré-termo morando apenas com a mãe. Com relação às características das famílias, houve diferença significativa entre os grupos no grau de escolaridade dos pais, com maiores níveis alcançados entre as mães do grupo a termo. 
Garcia NBC, et al. Coordenação fina e escrita de crianças de 6 a 9 anos. Rev Ter Ocup Univ São Paulo. 2015 maio/ago.;26(2):166-75.

\section{Análise da coordenação, destreza motora e escrita por avaliação direta da criança}

Não houve diferença significativa entre os grupos $(\mathrm{p}=0,23)$ na preferência manual, observada nas provas motoras e de escrita, com predomínio de preferência manual pela mão direita $(78,57 \%$ dos pré-termos e $89,29 \%$ dos a termo), nem com relação ao padrão de preensão e tipo de letra mantida durante as provas de escrita. Entre os pré-termos, $57,14 \%$ utilizaram letra cursiva; $21,43 \%$, letra bastão; e 21,43\% misturaram os dois padrões de letra; já entre os a termo, $46,43 \%$ utilizaram letra cursiva; $32,14 \%$, letra bastão; e $21,43 \%$ mesclaram os dois padrões de letra.
Quanto ao desempenho motor na ACOORDEM (Tabela 2), não foram encontradas diferenças significativas entre os grupos em nenhum item, no entanto o grupo pré-termo gastou mais tempo e cometeu mais erros em alguns itens, especialmente nos de recorte, mas a diferença não atingiu significância estatística. Quanto à escrita, foi encontrada diferença entre os grupos apenas no número de letras corretas na cópia do alfabeto, mas, novamente, o grupo pré-termo tendeu a pior desempenho na maioria dos itens avaliados. A análise dos dados do grupo pré-termo indica correlação significativa (Spearman) entre os escores totais de escrita - cópia do alfabeto e de sentença - e a pontuação em algumas provas de coordenação fina e destreza manual da ACOORDEM (Tabela 3).

Tabela 2 - Comparação entre os grupos nas provas de coordenação fina, destreza motora e escrita da ACOORDEM

\begin{tabular}{|c|c|c|c|c|c|}
\hline \multirow{2}{*}{ Subáreas e itens da ACOORDEM } & \multicolumn{2}{|c|}{ Pré-termo } & \multicolumn{2}{|c|}{ A termo } & \multirow{2}{*}{ Valor de p* } \\
\hline & MÉDIA ( \pm DP) & Mediana & MÉDIA ( $( \pm$ DP) & Mediana & \\
\hline \multicolumn{6}{|c|}{ BRINCANDO COM AS MÃOS (Tempo em segundos) } \\
\hline Colocar pinos na tábua (mão preferida) & $13,39( \pm 2,20)$ & 13,00 & $13,54( \pm 2,49)$ & 14,00 & 0,784 \\
\hline Colocar pinos na tábua (mão não preferida) & $15,14( \pm 2,56)$ & 15,00 & $15,39( \pm 2,75)$ & 15,00 & 0,869 \\
\hline Mudar pinos de fileira & $18,92( \pm 5,09)$ & 18,00 & $19,33( \pm 3,79)$ & 19,00 & 0,550 \\
\hline Colocar moedas no cofre (mão preferida) & $10,14( \pm 2,19)$ & 10,00 & $10,00( \pm 2,19)$ & 10,50 & 0,967 \\
\hline Colocar moedas no cofre (mão não preferida) & $12,37( \pm 3,53)$ & 12,00 & $11,21( \pm 2,73)$ & 12,00 & 0,222 \\
\hline Prova polegar-dedos (mão preferida) & $8,57( \pm 2,04)$ & 8,00 & $7,64( \pm 2,32)$ & 7,00 & 0,081 \\
\hline Prova polegar-dedo (mão não preferida) & $9,07( \pm 2,32)$ & 7,00 & $8,32( \pm 2,71)$ & 8,00 & 0,430 \\
\hline Distribuir 20 cartas & $23,07( \pm 9,45)$ & 19,50 & $19,96( \pm 7,06)$ & 19,00 & 0,264 \\
\hline Pesponto & $44,25( \pm 20,05)$ & 41,50 & $37,26( \pm 11,19)$ & 36,00 & 0,102 \\
\hline \multicolumn{6}{|c|}{ BRINCANDO DE DESENHAR (Número de erros) } \\
\hline Traçado reto & 0,00 & 0,00 & $0,04( \pm 0,19)$ & 0,00 & 0,317 \\
\hline Traçado curvo & $0,29( \pm 0,81)$ & 0,00 & $0,29( \pm 1,08)$ & 0,00 & 0,445 \\
\hline Trilha borboleta & $2,14( \pm 3,04)$ & 1,00 & $1,50( \pm 2,47)$ & 0,00 & 0,225 \\
\hline Cópia de figuras & $5,32( \pm 2,00)$ & 5,00 & $5,89( \pm 1,75)$ & 6,50 & 0,306 \\
\hline \multicolumn{6}{|c|}{ BRINCANDO DE RECORTAR (Número de erros) } \\
\hline Recorte reto & $1,19( \pm 2,47)$ & 0,00 & $0,27( \pm 1,00)$ & 0,00 & 0,144 \\
\hline Recorte quadrado & $4,04( \pm 4,50)$ & 3,00 & $2,22( \pm 2,85)$ & 1,00 & 0,065 \\
\hline Recorte círculo & $3,93( \pm 4,52)$ & 2,40 & $2,42( \pm 3,28)$ & 1,00 & 0,187 \\
\hline Recorte gato & $9,11( \pm 7,74)$ & 6,50 & $8,57( \pm 11,95)$ & 2,00 & 0,132 \\
\hline \multicolumn{6}{|l|}{ BRINCANDO DE ESCREVER } \\
\hline \multicolumn{6}{|l|}{ Cópia do alfabeto } \\
\hline Tempo gasto na cópia do alfabeto (seg.) & $91,11( \pm 40,27)$ & 84,00 & $102,75( \pm 43,72)$ & 90,50 & 0,317 \\
\hline Número total de letras escritas (26 letras) & $23,29( \pm 5,91)$ & 26,00 & $24,86( \pm 6,64)$ & 26,00 & 0,425 \\
\hline Total de letras corretas & $21,00( \pm 6,14)$ & 23,50 & $23,07( \pm 5,39)$ & 26,00 & 0,019 \\
\hline Velocidade - letras escritas por minuto & $15,87( \pm 7,47)$ & 15,40 & $15,37( \pm 6,82)$ & 13,60 & 0,844 \\
\hline Cópia de letras corretas e legíveis $\left(32^{\mathrm{a}}\right)$ & $30,39( \pm 2,04)$ & 31,00 & $31,18( \pm 1,09)$ & 32,00 & 0,128 \\
\hline Escore total de cópia do alfabeto & $44,86( \pm 3,03)$ & 45,00 & $46,29( \pm 2,16)$ & 47,00 & 0,061 \\
\hline \multicolumn{6}{|l|}{ Cópia de sentença } \\
\hline Tempo gasto na cópia da sentença (seg.) & $58,54( \pm 45,58)$ & 51,50 & $83,64( \pm 74,14)$ & 62,00 & 0,356 \\
\hline Número total de letras escritas (45 letras) & $33,25( \pm 19,64)$ & 45,00 & $34,43( \pm 18,74)$ & 45,00 & 0,916 \\
\hline Total de letras corretas & $32,11( \pm 18,09)$ & 43,00 & $32,39( \pm 18,09)$ & 42,50 & 0,712 \\
\hline Velocidade - letras escritas por minuto & $30,22( \pm 23,87)$ & 26,50 & $26,61( \pm 22,58)$ & 20,65 & 0,604 \\
\hline Cópia de letras corretas e legíveis $\left(36^{\text {a }}\right)$ & $23,68( \pm 15,46)$ & 31,50 & $25,75( \pm 14,16)$ & 32,50 & 0,525 \\
\hline Escore total de cópia de sentença & $36,75( \pm 20,19)$ & 47,00 & $38,21( \pm 19,47)$ & 48,00 & 0,565 \\
\hline Nota da professora para escrita (0 a 10) & $6,18( \pm 2,65)$ & 7,00 & $6,89( \pm 1,87)$ & 7,00 & 0,372 \\
\hline
\end{tabular}

Nota: DP = desvio padrão; ${ }^{\mathrm{a}=}$ escore máximo; *Teste de Mann Whitney 
Garcia NBC, et al. Coordenação fina e escrita de crianças de 6 a 9 anos. Rev Ter Ocup Univ São Paulo. 2015 maio/ago.;26(2):166-75.

Tabela 3 - Correlação (Spearman) entre a pontuação total na escrita - cópia de alfabeto e de sentença - e pontuação nos itens motores e questionários

\begin{tabular}{|c|c|c|c|c|}
\hline & Total Alfabeto & Valor de $p$ & Total Sentença & Valor de $p$ \\
\hline Colocar pinos na tabua - mão preferida & $-0,483$ & 0,009 & $-0,611$ & 0,001 \\
\hline Colocar pinos na tabua - mão não preferida & $-0,105$ & 0,594 & $-0,292$ & 0,131 \\
\hline Mudar pinos fileira - somente mão preferida & 0,123 & 0,557 & $-0,178$ & 0,393 \\
\hline Pesponto & $-0,20$ & 0,35 & $-0,284$ & 0,179 \\
\hline Colocar moedas cofre - mão não preferida & 0,198 & 0,322 & 0,101 &, 615 \\
\hline Colocar moedas cofre - mão preferida & 0,244 & 0,21 & $-0,013$ & 0,95 \\
\hline Distribuir cartas & $-0,126$ & 0,523 & $-0,464$ & 0,013 \\
\hline Polegar - dedos mão preferida & $-0,026$ & 0,894 & $-0,191$ & 0,331 \\
\hline Polegar - dedos mão não preferida & $-0,006$ & 0,975 & $-0,271$ & 0,163 \\
\hline Recorte linha reto & $-0,408$ & 0,034 & $-0,325$ & 0,098 \\
\hline Recorte quadrado & $-0,486$ & 0,009 & $-0,602$ & 0,001 \\
\hline Recorte círculo & $-0,306$ & 0,146 & $-0,417$ & 0,043 \\
\hline Recorte formato de gato & $-0,475$ & 0,011 & $-0,570$ & 0,002 \\
\hline Traçado - trilha em linha reta & $-0,321$ & 0,096 & $-0,487$ & 0,009 \\
\hline Traçado - trilha curva & $-0,281$ & 0,148 & $-0,366$ & 0,056 \\
\hline Traçado - trilha da borboleta & $-0,583$ & 0,001 & $-0,594$ & 0,001 \\
\hline Copia de figuras (8 figuras) & 0,323 & 0,093 & 0,544 & 0,003 \\
\hline Pontuação Total no DCDQ & 0,158 & 0,421 & 0,354 & 0,064 \\
\hline $\begin{array}{l}\text { Questionário ACOORDEM para pais - área de } \\
\text { mobilidade }\end{array}$ & 0,043 & 0,827 & 0,25 & 0,199 \\
\hline $\begin{array}{l}\text { Questionário ACCORDEM para pais - área de } \\
\text { habilidades para A.V.D. }\end{array}$ & 0,137 & 0,487 & 0,489 & 0,008 \\
\hline $\begin{array}{l}\text { Questionário ACOORDEM para pais - área do } \\
\text { papel do estudante }\end{array}$ & $-0,373$ & 0,051 & $-0,514$ & 0,005 \\
\hline $\begin{array}{l}\text { Questionário ACOORDEM para pais - área de } \\
\text { comportamento, hábitos e rotinas }\end{array}$ & 0,194 & 0,324 & 0,151 & 0,443 \\
\hline $\begin{array}{l}\text { Questionário ACCORDEM para professores - área } \\
\text { de habilidades motoras }\end{array}$ & 0,212 & 0,279 & 0,334 & 0,083 \\
\hline $\begin{array}{l}\text { Questionário ACCORDEM para professores - área } \\
\text { de comportamento }\end{array}$ & 0,505 & 0,006 & 0,550 & 0,002 \\
\hline
\end{tabular}

No DCDQ-Brasil, $\quad 10,71 \%$ dos pré-termos apresentaram sinais de problemas de coordenação motora global, enquanto $100 \%$ dos a termo apresentaram desenvolvimento motor típico. Apesar dessa pequena diferença entre os grupos, o teste de Fisher não foi significativo $(\mathrm{p}=0,236)$, no entanto crianças de menor peso ao nascimento tenderam a apresentar pontuações mais baixas no questionário.

No ETDAH, não foram encontradas diferenças significativas entre os grupos para sinais de problemas nas quatro áreas do instrumento - déficit de atenção, hiperatividade/impulsividade, problemas de aprendizagem e comportamento antissocial - mas houve maior número de crianças pré-termo com indicativo de mais problemas que a média e possível transtorno nas subáreas "problemas de aprendizagem" (pré-termo $=21,43 \%$, a termo $=$ $10,71 \%$ ) e "déficit de atenção" (pré-termo $=39,28 \%$, a termo $=17,86 \%$ ).

Nos questionários da ACOORDEM respondidos pelos pais e professores, houve diferença significativa entre os grupos, com pior desempenho para o grupo pré-termo em várias áreas (Tabela 4). 
Garcia NBC, et al. Coordenação fina e escrita de crianças de 6 a 9 anos. Rev Ter Ocup Univ São Paulo. 2015 maio/ago.;26(2):166-75.

Tabela 4 - Comparação entre os grupos nos questionários para pais e professores da ACOORDEM

\begin{tabular}{|c|c|c|c|c|c|}
\hline \multirow{2}{*}{ QUESTIONÁRIOS } & \multicolumn{2}{|c|}{ PRÉ-TERMO } & \multicolumn{2}{|c|}{ A TERMO } & \multirow{2}{*}{$\begin{array}{l}\text { Valor } \\
\text { de } p^{*}\end{array}$} \\
\hline & MÉDIA ( \pm DP) & Mediana & MÉDIA ( \pm DP) & Mediana & \\
\hline \multicolumn{6}{|l|}{ QUESTIONÁRIO PROFESSORES } \\
\hline Escala motora & $61,11( \pm 10,87)$ & 63,50 & $69,68( \pm 8,23)$ & 70,50 & $\mathbf{0 , 0 2 7}$ \\
\hline Escala de comportamento & $29,11( \pm 8,08)$ & 29,00 & $33,93( \pm 6,71)$ & 36,50 & 0,024 \\
\hline \multicolumn{6}{|l|}{ QUESTIONÁRIO DE PAIS } \\
\hline $\begin{array}{l}\text { Mobilidade e habilidade para participar de jogos } \\
\text { e brincadeiras }\end{array}$ & $56,93( \pm 3,310)$ & 57,50 & $58,11( \pm 2,97)$ & 59,00 & 0,147 \\
\hline $\begin{array}{l}\text { Habilidades para desempenhar atividades de } \\
\text { vida diária }\end{array}$ & $52,32( \pm 5,026)$ & 52,50 & $56,07( \pm 2,07)$ & 56,00 & 0,002 \\
\hline Habilidades relacionadas ao papel do estudante & $35,46( \pm 4,558)$ & 36,50 & $35,61( \pm 5,05)$ & 37,00 & 0,613 \\
\hline Comportamento, hábitos e rotinas & $47,00( \pm 6,896)$ & 47,50 & $51,71( \pm 2,87)$ & 51,00 & 0,007 \\
\hline
\end{tabular}

Nota: DP = desvio padrão; *Teste Mann Whitney

\section{DISCUSSÃO}

Embora não tenham sido encontradas diferenças significativas no desempenho motor fino e na escrita entre os escolares nascidos pré-termo e a termo, os dados merecem discussão detalhada, pois revelam tanto aspectos da assistência ao recém-nascido pré-termo em cidades de pequeno porte no interior do país, como permitem fazer inferências sobre o desenvolvimento motor e cuidados necessários na educação essas crianças.

Inicialmente chama a atenção a falta de orientações específicas aos pais acerca da prematuridade após a alta hospitalar, com mais de $85 \%$ das mães relatando não terem recebido qualquer informação acerca do nascimento prematuro e suas possíveis implicações e apenas uma das mães relatou que seu filho teve algum acompanhamento especializado após o nascimento devido à prematuridade. Este dado ganha relevância quando se considera que a amostra incluiu aproximadamente $100 \%$ das crianças prematuras nascidas no município, conforme os critérios de inclusão.

Com relação a aspectos motores, considerandose as provas motoras e de escrita, não foi encontrada diferença significativa entre os grupos em nenhum dos itens avaliados, exceto na quantidade de letras do alfabeto escritas corretamente (Tabela 3), na qual o grupo pré-termo teve pior desempenho. Apesar de a diferença entre grupos não ter alcançado significância estatística, o escore total de provas de escrita, tanto para cópia do alfabeto quanto para da sentença, foi inferior entre o grupo pré-termo.

Nota-se tendência a maior lentidão e maior número de erros nas provas de destreza manual, em uma prova de traçado e em todas as provas de recorte no grupo pré-termo, mas sem significância estatística. Nas provas de escrita, as crianças pré-termo tenderam a ser mais rápidas, mas com menor acuidade, como indicado pelo menor número total de letras do alfabeto escritas corretamente. Os resultados dão suporte parcial aos achados na literatura ${ }^{14}$ sobre comprometimento da escrita e seus subcomponentes, de coordenação fina e controle viso-motor, entre os pré-termos; no entanto o tamanho pequeno da amostra do presente estudo possivelmente limitou as possibilidades de se atingir significância estatística em maior número de variáveis. É importante enfatizar que a amostra estudada foi constituída por pré-termos limítrofes e moderados, nos quais não se espera déficits motores acentuados. Deve-se observar, ainda, que como as professoras foram instruídas a ajudar a recrutar pares com nível de funcionamento semelhante, elas possivelmente indicaram crianças com padrão de desempenho escolar similar ao par pré-termo, o que contribuiu para que os grupos fossem homogêneos também nas provas de desempenho. Em futuros estudos recomendamos que o recrutamento para pareamento seja realizado por sorteio.

Quanto à escrita, especificamente, um fator que parece ter influenciado o desempenho de ambos os grupos foi a forma com que essa habilidade é ensinada nas escolas investigadas. Foi observado que ambos os grupos tiveram grande dificuldade nas tarefas de escrita, dificuldades estas além do esperado, uma vez que a amostra compreendia escolares de 6 a até 9 anos, que tiveram muita dificuldade para lembrar todas as letras do alfabeto e copiar uma sentença simples. Inicialmente, quando lhes foi pedido para realizar as provas com letra cursiva, $42,86 \%$ dos prétermos e $53,57 \%$ dos a termo não foram capaz de utilizar ou manter o padrão de letra cursiva, sendo então pontuado 
qualquer tipo de letra compreensível. Ainda assim, o número médio de letras do alfabeto escritas corretamente variou entre 21 (pré-termo) a 22 (a termo) letras, para um total de 26 letras. Além disso, $25 \%$ dos pré-termos e $21 \%$ dos nascidos a termo declaram não saber escrever e, por isso, se recusaram a copiar uma sentença simples. Dada a dificuldade das crianças, os critérios de escore dos itens do teste tiveram que ser adaptados para permitir a pontuação da amostra, uma vez que na ACOORDEM se pontua apenas letras cursivas, e no presente estudo tivemos que considerar qualquer tipo de letra identificável. Esses ajustes possivelmente influenciaram a capacidade do teste para discriminar diferenças de desempenho entre os grupos.

O pobre desempenho na escrita da amostra como um todo pode estar relacionado à qualidade do ensino nas escolas municipais, que talvez não ofereçam suporte e estímulo adequados para a boa aquisição da escrita. Essa hipótese se baseia em dados da avaliação nacional da qualidade do ensino, realizada pelo INEP em 2011, por meio da Prova Brasil, que indicou que nos primeiros anos do ensino fundamental os resultados municipais foram inferiores aos dados de referência ao estado de São Paulo $^{21}$. Outra tendência que foi possível observar é que, entre os escolares da escolar particular, o desempenho nas provas de escrita, em ambos os grupos, foi mais consistente, sendo observada maior facilidade para escrever.

Assim como discutido por outros autores ${ }^{22,23}$, foram encontradas correlações significativas entre o escore total nas provas de escrita e a coordenação motora (Tabela 3), principalmente nas provas motoras mais difíceis, como colocar pinos na tábua com a mão preferida, recortar o quadrado e um gato e traçar uma borboleta, o que dá suporte à compreensão da escrita como uma atividade motora complexa.

Com relação aos questionários, iniciando pelas informações dadas pelos professores, chama atenção o fato de apenas o questionário de comportamento ter tido correlação positiva com a escrita, com crianças com melhor comportamento obtendo melhor pontuação nas duas provas de escrita. No entanto não houve correlação com a subescore motor do questionário, que inclui itens de escrita. Isso sugere que as professoras possivelmente estão mais atentas a questões de comportamento do que de qualidade da escrita. No questionário de pais houve correlação significativa entre habilidade para copiar sentenças e o desempenho nas atividades de vida diárias, que compartilham aspectos relacionados à destreza manual. Por outro lado, observa-se correlação negativa entre a pontuação cópia de sentença e as habilidades relacionadas ao papel de estudante, parte do questionário que inclui vários itens de escrita. Analisando os dados, foi observado que os pais pontuaram muito positivamente as habilidades dos filhos para fazer as tarefas escolares, enquanto os escores nas provas de escrita não foram tão bons. Esse dado sugere que os pais não mostraram boa habilidade para pontuar o desempenho dos filhos nas tarefas escolares, o que pode ser explicado pelo baixo nível de escolaridade dos pais das crianças pré-termo. Recomenda-se, portanto, que informação dos pais sobre o desempenho em atividades escolares seja sempre completado por dados coletados com os professores.

É interessante observar que, embora não tenham sido encontradas diferenças significativas entre os grupos no desempenho motor e na escrita, como medido pelos itens observacionais da ACOORDEM, pais e professores parecem notar dificuldades nas crianças pré-termo, como sinalizado pelas diferenças significativas em duas áreas do questionário de pais (AVD e hábitos e rotinas) e nas duas áreas do questionário de professores (motor e comportamento). Talvez a avaliação motora direta da criança conforme os critérios apenas da ACCORDEM não seja suficientemente sensível para detectar as diferenças motoras sutis que os pais e professores aparentemente conseguem detectar nos escolares em casa e na escola, uma vez que os déficits motores das crianças podem não se concentrar apenas na função manual.

Com relação à prevalência de sinais de TDA/H, apesar de não ter alcançado significância estatística, houve maior percentual de ocorrência de mais problemas do que a média nas quatro áreas de avaliação (hiperatividade, problemas de aprendizagem, comportamento antissocial e déficit de atenção) no grupo pré-termo. Outros autores ${ }^{10 ; 24}$ reportam prevalências mais altas de problemas de atenção e coordenação motora entre crianças nascidas pré-termo, mas em amostras de menor peso ao nascimento e idade gestacional.

As diferenças encontradas entre os grupos foram sutis e muitas não atingiram significância, possivelmente devido ao pequeno porte da cidade estudada, mesmo que tenha compreendido aproximadamente $100 \%$ dos prétermos nascidos no período em estudo e a heterogeneidade da amostra no que diz respeito à idade dos escolares (6 a 9 anos), que gerou grandes desvios padrão, especialmente no grupo pré-termo. Recomenda-se que nos próximos estudos se busque maior homogeneidade intragrupos, com recrutamento de grupos representativos por faixa de idade e aplicação da ACOORDEM na íntegra juntamente com um teste de coordenação motora validado, de forma a investigar se a ACOORDEM é útil para identificar problemas leves de coordenação motora. 
Garcia NBC, et al. Coordenação fina e escrita de crianças de 6 a 9 anos. Rev Ter Ocup Univ São Paulo. 2015 maio/ago.;26(2):166-75.

Os pontos fortes do trabalho relacionam-se com o fato de a amostra compreender aproximadamente $100 \%$ do possível no município estudado, à metodologia de localização dos pré-termos por meio de vários mecanismos de localização das famílias e ao fato de a aplicação da ACOORDEM somente ter sido feita após treinamento e aplicação conjunta para confiabilidade. As limitações do estudo são o número reduzido de participantes e o modo de seleção do grupo a termo por indicação da professora, ao invés de sorteio.

\section{REFERÊNCIAS}

1. Ayache MG, Corintio MN. Considerações sobre o desenvolvimento motor do prematuro. Temas Desenvol. 2003;12(71):5-9.

2. Rodrigues MCC, Melo RR, Silva KS, Carvalho ML. Desenvolvimento cognitivo de prematuros à idade escolar: proposta de modelo hierarquizado para investigação dos fatores de risco. Cad Saúde Pública. 2011;27(6):1154-64. DOI: http://dx.doi.org/10.1590/ S0102-311X2011000600012.

3. Petrini JR, Dias T, McCormick MC, Massolo ML, Geen NS, Escobar, GJ. Increased risk of adverse neurological development for late preterm infants. J Pediatr. 2009;154(2):169-76. DOI: 10.1016/j.jpeds.2008.08.020.

4. Van Batenburg-Eddes TV, de Groot L, Arends L, de Vries A, Moll HA, Steegers EA, Hofman A, Jaddoe VW, Verhulst FC, Tiemeier H. Does gestational duration within the normal range predict infant neuromotor development? Early Hum Dev. 2008;84(10):659-65. DOI: 10.1016/j. earlhumdev.2008.04.007.

5. Magalhães LC, Catarina PW, Barbosa VM, Mancini MC, Paixão ML. Estudo comparativo sobre o desempenho perceptual e motor na idade escolar em crianças nascidas pré-termo e a termo. Arq Neuropsiquiatr. 2003;61(2A):250-5. Disponível em: http://www.scielo.br/pdf/anp/ v61n2A/15691.pdf

6. Treyvauld K, Anderson VA, Howard K, Bear M, Hunt RW, Doyle LW, Inder TE, Woodward L, Anderson PJ. Parenting behavior is associated with the early neurobehavioral development of very preterm children. Pediatrics. 2009;123(2):555-61. DOI: 10.1542/peds.2008-0477.

7. Foster-Cohen SH, Friesen MD, Champion PR, Woodward LJ. High prevalence/low severity langrage delay in preschool children born very preterm. J Dev Behav Pediatr. 2010;31(8):658-67. DOI: 10.1097/ DBP.0b013e3181e5ab7e.
As crianças nascidas pré-termo parecem ter maior dificuldade motora fina e na escrita, além de apresentar mais sinais de transtornos comportamentais, mesmo dentro da faixa de pré-termos limítrofes. Assim, devem-se oferecer orientações às mães quanto às possíveis consequências da prematuridade, fornecer programas de acompanhamento ao desenvolvimento e atenção especial na escola para que essas crianças recebam um olhar mais atencioso ao seu desenvolvimento.

8. Edwards J, Berube M, Erlandson K, Haug S, Johnstone H, Meagher M, Sarkodee-Adoo S, Zwicker JG. Developmental coordination disorder in school-aged vhildren born very preterm and/or at very low birth weight: a systematic review. J Dev Behav Pediatr. 2011;32(9):678-87. DOI: 10.1097/DBP.0b013e31822a396a.

9. Howe TH, Sheu CF, Wang TN, Hsu YW, Wang LW. Neuromotor outcomes in children with very low birth weight at 5 yrs of age. Am J Phys Med Rehabil. 2011;90(8):667-80. DOI: 10.1097/PHM.0b013e31821a703f.

10. McGrath MM, Sullivan M, Devin J, Fontes-Murphy M, Barcelos S, De Palma JL, Faraone S. Early precursors of low attention and hyperactivity in a preterm sample at age four. Issues Compr Pediatr Nurs. 2005;28(1):1-15. DOI: $10.1080 / 01460860590913945$.

11. Zwicker JG, Yoon SW, Mackay M, Petrie-Thomas J, Rogers M, Synnes AR. Perinatal and neonatal predictors of developmental coordination disorder in very low birth weight children. Arch Dis Child. 2013;98(2)118-22. DOI: 10.1136/archdischild-2012-302268.

12. Guarini A, Sansavini A, Fabbri C, Savini S, Alessandroni R, Faldella G, Karmiloff-Smith A. Long-term effects of preterm birth on language and literacy at eight years. J Child Lang. 2010;37(4):865-85. DOI: 10.1017/ S0305000909990109.

13. Roberts G, Lim J, Doyle LW, Anderson PJ. High rates of school readiness difficulties at 5 years of age in very preterm infants compared with term controls. J Dev Behav Pediatr. 2011;32(2):117-24. DOI: 10.1097/DBP.0b013e318206d5c9.

14. FederKP, Majnemer A, Bourbonnais D, Platt RM, Blayney M, Synnes A. Handwriting performance in preterm children compared with terms peers at age 6 to 7 years. Dev Med Child Neurol. 2005;47(3):163-70. DOI: 10.1111/j.1469-8749.2005.tb01110.x.

15. Daly CJ, Kelley GT, Kraus A. Relationship between visual-motor integration an handwriting skiils of children in kindergarten: A modified replication study. Am J OccupTher. 2003;57(4):459-62. DOI:10.5014/ajot.57.4.459. 
Garcia NBC, et al. Coordenação fina e escrita de crianças de 6 a 9 anos. Rev Ter Ocup Univ São Paulo. 2015 maio/ago.;26(2):166-75.

16. Magalhães LC, Resende MB, Cardoso AM, Galvão BAP, Maor FMOM. Relação entre destreza manual e legibilidade da escrita em crianças: estudo piloto. Rev Ter Ocup Univ São Paulo. 2011;22(2):127-35. DOI: http://dx.doi.org/10.11606/ issn.2238-6149.v22i2p127-135

17. Lacerda TTB. Estudo sobre a validade dos questionários de pais e professores da ACOORDEM: avaliação da coordenação e destreza motora [Dissertação]. Belo Horizonte: Escola de Educação Física, Fisioterapia e Terapia Ocupacional, Universidade Federal de Minas Gerais; 2006.

18. Cardoso AA, Magalhães LC. Análise da validade de critérios da avaliação da coordenação e destreza motora ACOORDEM para crianças de 7 a 8 anos de idade. Rev Bras Fisioter. 2011;16(1):16-22. DOI: http://dx.doi.org/10.1590/ S1413-35552012000100004.

19. Cardoso AA, Magalhães LC, Lacerda TTB, Andrade PMO. Relação entre a avaliação da coordenação e destreza motora (ACOORDEM) e a Classificação Internacional de Funcionalidade, Incapacidade e Saúde (CIF). Fisioter Mov. 2012;25(1):31-45. DOI: http://dx.doi.org/10.1590/S010351502012000100004 .

20. Mazer EP, Della Barbba PC. Identificação de sinais de transtornos de desenvolvimento da coordenação em crianças de três a seis anos e possibilidades de atuação da terapia ocupacional. Rev Ter Ocup Univ São Paulo. 2010;21(1): 74-82. DOI: http://dx.doi.org/10.11606/issn.2238-6149. v21i1p74-82.

21. Instituto Nacional de Estudos e Pesquisas Educacionais Anísio Teixeira (INEP) [Citado 28 out. 2013]. Disponível em: www.sistemasprovabrasil2.inep.gov.br.

22. Calvo AP, Pellegrini AM, Higara CY. Estariam as dificuldades de escrita associadas a dificuldades motoras? Col Pesq Educ Física. 2007;16:275-82. Disponível em: http://www.fontouraeditora.com.br/periodico/vol-6/ Vol6n1-2007/Vol6n1-2007-pag-275a282/Vol6n1-2007pag-275a282.pdf.

23. Feder KP, Majnemer A. Handwriting development, competency and intervention. Dev Med Child Neurol. 2007; 49(4):312-7. DOI: 10.1111/j.1469-8749.2007.00312.x.

24. Oliveira GE, Magalhães LC, Salmela LFT. Relationship between very low birth weight, environmental factors, and motor and cognitive development of children of 5 and 6 years old. Rev Bras Fisioter. 2011;15(2):138-45. DOI: http://dx.doi.org/10.1590/S1413-35552011000200009. 